\title{
Ways to resolve Selleri's paradox
}

\author{
Klaus Kassnel® \\ Institut für Theoretische Physik, \\ Otto-von-Guericke-Universität Magdeburg, Germany
}

(Dated: 26 June 2012)

\begin{abstract}
Selleri's paradox, based on an analysis of rotating frames, appears to show that the speed of light in an inertial system is not normally isotropic. This in turn seems at odds with the second postulate of special relativity requiring a universal light speed in inertial systems. First, it is demonstrated how to circumvent Selleri's argument using Einstein synchronization in rotating frames. Then the nature of Selleri's result is exposed: it simply corresponds to the adoption of a synchronization procedure different from Einstein's. In this scheme, anisotropic one-way speeds of light by no means contradict special relativity.
\end{abstract}

PACS numbers: $03.30 .+\mathrm{p} ; 04.20 . \mathrm{Cv}$ 04.20.Gz

Keywords: Special relativity, Selleri paradox, clock synchronization, conventionality of simultaneity

\section{INTRODUCTION}

Special relativity is a most successful theory. Many peculiar predictions can be derived from it, some very counterintuitive. Nevertheless, when tested experimentally, they invariably turn out to be true. One of the best-known phenomena is time dilation, which has been demonstrated in many experiments, $\stackrel{1-5}{-5}$ including both measurements of the life time of decaying particles (muons) and direct use of atomic clocks. A famous realization of the twin paradox is the Hafele-Keating experiment ${ }^{6,7}$ employing airplane borne traveling clocks. The clock hypothesis (independence of time dilation of acceleration) has been verified using muons that were subject to accelerations of $10^{18} \mathrm{~g}$ in a storage ring. $\frac{8}{-}$ Isotropy of the round-trip speed of light has been shown to be true with everincreasing accuracy ${ }^{9-11}$ There have been explicit tests of the isotropy of space ${ }^{12,13}$ and of Lorentz invariance ${ }^{14,15}$ and there is a huge amount of literature on the tight limits of possible violations of Lorentz invariance. ${ }^{16}$ The independence of the speed of light of the velocity of its source has also been explicitly addressed.17,18 Modern synchrotrons would not work, if the speed-dependent change of inertia of electrons ${ }^{19}$ was not taken into account.

Many of the particular effects of special relativity have been demonstrated separately $16,20,21$ An exception seems to be Lorentz contraction, often quoted as not having been observed directly. It is however clear that even the MichelsonMorley experiment ${ }^{9}$ is an indirect proof of Lorentz contraction, if one accepts standard arguments ${ }^{22}$ as to why there can be neither contraction nor expansion in the direction perpendicular to the velocity. Then length contraction parallel to it is needed to explain the null result of the experiment in inertial frames where the interferometer is not at rest.

Despite this satisfactory status regarding the experimental basis of special relativity, which has become one of the pillars of modern physics, ${ }^{21}$ other fundamental theories with a similar level of maturity such as, say, statistical mechanics seem to have had fewer acceptance problems. ${ }^{23}$ Adverse reactions to special relativity were not due to a particularly demanding mathematical framework. As long as descriptions are set up in inertial systems, the mathematics of the theory remains simple. Rather, they have to do with the fact that the theory is an imposition at the conceptual level. For members of a species whose survival at times depended on collective welltimed actions, the idea of simultaneity not being the same for everybody definitely had an insane connotation. Even now, such an idea is difficult to convey in everyday life, where it is important that musicians in a band or players in a soccer team agree on just when things happen in their environment to which they have to react. ${ }^{24}$ The speed of light is so large that the relativity of simultaneity could go unnoticed for almost all of human history.

Most of the paradoxes invoked to indicate flaws in special relativity can be resolved by pointing out inadvertent negligence of the fact that (spatially separated) events regarded as simultaneous by one observer are not necessarily simultaneous for another observer. This is true for kinematic paradoxes, whereas apparent contradictions involving forces, such as the submarine paradox, ${ }^{25,26}$ are more difficult to solve.

As Will notes, ${ }^{21}$ special relativity is rarely challenged today. However, even now there are some authors who believe special relativity to fail, either in accounting for the physics in rotating frames ${ }^{27,28}$ or more generally, due to conceptual flaws. ${ }^{29}$ Selleri, who certainly understands the theory well, invented his paradox, introduced in Sec. II] hoping to deprive special relativity of its foundations. The purpose of this paper is to first resolve the paradox within the framework of a standard approach (Sec. III). Second, a less conventional (and more elegant) solution is given in Sec. III that highlights the role of synchronization conventions within the theory. A few conclusions summarize what we may learn from Selleri's objection (Sec. IV].

\section{THE SAGNAC EFFECT AND SELLERI'S PARADOX}

Consider a disk rotating at constant angular velocity $\omega$ and an observer $O_{d}$ stationary on the disk at radius $R$. This observer sends two light signals around the disk in opposite directions along the circle $r=R$. What time will $O_{d}$ measure until the return of each signal? First consider the description by an inertial observer $C_{0}$ at the center of the disk. Light runs around the disk at velocity $R \mathrm{~d} \varphi / \mathrm{d} t= \pm c$, the observer $O_{d}$ at $R$ moves at $\mathrm{d} \varphi / \mathrm{d} t=\omega$, both motions lead to linear time laws, so the time spans $T_{ \pm}$for the signals to return to $O_{d}$ are given by $\pm c T_{ \pm}=R \omega T_{ \pm} \pm 2 \pi R$, hence

$$
T_{ \pm}=\frac{2 \pi R}{c \mp \omega R} \text {. }
$$

The local time of $O_{d}$ runs slower than the time of $C_{0}$ by the time dilation factor $1 / \gamma=\left(1-\omega^{2} R^{2} / c^{2}\right)^{1 / 2}$, so for him the 
light signals take the times

$$
\tau_{ \pm}=\left(1-\frac{\omega^{2} R^{2}}{c^{2}}\right)^{1 / 2} \frac{2 \pi R}{c \mp \omega R}=\frac{2 \pi R}{c}\left(\frac{1 \pm \frac{\omega R}{c}}{1 \mp \frac{\omega R}{c}}\right)^{1 / 2}
$$

in the forward (+) and backward (-) directions, respectively. Taking into account that for a disk observer the circumference of the coordinate line $r=R$ is $L^{\prime}=2 \pi R\left(1-\frac{\omega^{2} R^{2}}{c^{2}}\right)^{-1 / 2}$ as measured by standard rulers (which are Lorentz contracted, so more of them are needed to cover the $\operatorname{circle}^{30-32}$ ), we find the average speeds of light in the co- and counterrotating senses to be

$$
c_{ \pm}=\frac{2 \pi R}{\left(1-\frac{\omega^{2} R^{2}}{c^{2}}\right)^{1 / 2} \tau_{ \pm}}=\frac{c}{1 \pm \frac{\omega R}{c}} .
$$

It may surprise that light needs different times to orbit the disk in the two directions. This is the Sagnac effect and it was taken by its discoverer as a sign for a failure of special relativity ${ }^{33}$ Indeed, the result seems difficult to reconcile with a universal speed of light.

On top of this, Selleri constructed an interesting paradox arising from the Sagnac effect. ${ }^{27,29}$ First, he argued that the local speed of light along a circle must be equal to its average speed for symmetry reasons. All points on the circumference of the circle are physically equivalent, so any local observer $O_{l}$ there should definitely measure the same velocity of light along equally oriented tangents. With all speeds being equal, their average cannot take a different value. Second, the ratio of these speeds for the two tangential orientations is

$$
q \equiv \frac{c_{+}}{c_{-}}=\frac{c-\omega R}{c+\omega R} \neq 1
$$

which does not depend on $\omega$ and $R$ separately but only on their product $v=\omega R$. Imagine the radius $R$ going to infinity and the angular frequency of the disk going to zero in a way that keeps $v$ constant. The centripetal acceleration of a point at $r=R$ is $\omega^{2} R=v^{2} / R$ and approaches zero as $R$ is sent to infinity. Obviously, the system will then approach an inertial system, but $q=q(v)$ will remain unchanged. Hence we have constructed an inertial system, in which the speeds of light in the forward and backward directions are different! This may look like a proof that special relativity is wrong. Selleri's intention was indeed to provide such a proof.

It might be added that the mindset of this "proof" is entirely Newtonian. The symmetry argument asserts equality of the local speed of light with its average $c_{\mathrm{av}}$ over all the locations along the light path. (We omit the subscript \pm for brevity.) Because the whole situation is stationary, it may also be argued that this average is equal to the average along an actual light path, i.e., when the different locations are passed successively. It should not matter, when the speed of light is measured at a point. - But is this also the average speed measured by a single observer $O_{d}$ waiting for the signal to return? An appropriate expression for that average reads

$$
c_{\mathrm{av}}^{O}=\frac{L^{\prime}}{\tau}=\frac{1}{\tau} \oint \mathrm{d} s^{\prime}=\frac{1}{\tau} \int \frac{\mathrm{d} s^{\prime}}{\mathrm{d} t^{\prime}} \mathrm{d} t^{\prime}=\frac{1}{\tau} \int c_{l}\left(t^{\prime}\right) \mathrm{d} t^{\prime} .
$$

Here, $c_{l}\left(t^{\prime}\right)=\mathrm{d} s^{\prime} / \mathrm{d} t^{\prime}$ is the local speed of light as "seen" by $O_{d}$, which might be different from what a local observer $O_{l}\left(s^{\prime}\right)$ at the position $s^{\prime}$ sees. While on a disk rotating at constant angular velocity the distances of observers at rest on the disk will remain constant, ${ }^{34}$ the observer $O_{d}$ will, if he uses a frame of reference with axes that are parallel to those of an inertial system, note $O_{l}\left(s^{\prime}\right)$ to revolve around himself (i.e., $O_{d}$ ) with respect to these fixed axes. This can be measured with the help of a gyroscope. Should he then not expect $O_{l}\left(s^{\prime}\right)$ 's clock to suffer time dilation with respect to his own clock and would that not render $c_{l}$ (and $c_{\mathrm{av}}^{O}$ ) different from $c_{\mathrm{av}}$ ? One might also expect length contraction, but this objection can be discarded easily. $\mathrm{d} s^{\prime}$ is by definition the length element measured with a local ruler, so all observers along the circle will agree on local lengths by convention. In a Newtonian world, the issue would then be settled, because time is absolute, so if both observers agree on $\mathrm{d} s^{\prime}$, they have to agree on the local velocity. In a relativistic world, we need an argument why they should also agree on $\mathrm{d} t^{\prime}$.

Note that the two-way speed of light along our circle will still conform with Einstein's second postulate, even if we assume the local velocities to be $c_{+}$and $c_{-}$. Indeed, a light beam sent a distance $\Delta s$ to a mirror and reflected back, will take the round-trip time

$$
\Delta t=\frac{\Delta s}{c_{+}}+\frac{\Delta s}{c_{-}}=\frac{\Delta s}{c}\left(1+\frac{\omega R}{c}+1-\frac{\omega R}{c}\right)=\frac{2 \Delta s}{c},
$$

meaning that the average speed of light is $c$.

Contrary to Selleri's beliefs, $\stackrel{35}{2}$ a one-way velocity of light that is different from $c$ does not mean a breakdown of the first postulate of special relativity, if the latter is properly understood as a statement about physics and not one about the mathematical formulation thereof. Instead of saying that the form of physical laws is the same in all inertial systems, ${ }^{36}$ we may simply state that the outcome of any local physical experiment (i.e., an experiment not relying on observation of remote events) is the same in all inertial systems. This also clarifies the issue of independence of the first and second postulates. ${ }^{37}$

Before dealing with Selleri's paradox, let us see how the Sagnac effect itself can be explained from the point of view of local comoving observers. Each of these observers may be considered to be in an instantaneous local inertial frame of reference. Within these frames, clocks can be synchronized according to Einstein's prescription ${ }^{38}$ We may then conclude from the second postulate referred to the two-way velocity of light that the one-way velocity of light also has to be $c .^{39}$

In order to combine these local observations into a global description, it is useful to consider a sequence of local Minkowski diagrams, intersect them with the cylindrical world sheet of the circle, cut this open and roll it out onto a plane. The resulting picture is shown in Fig. 1. Because the rotation speed $v=\omega R$ is the same at all points considered, the spatial axes of all local observers are parallel and can be made to coincide, so that our Minkowski diagram contains only two sets of axes, one for an observer $C$ at rest with respect to the center of the disk (but located at $r=R$ ) and one corresponding to all of the locally comoving observers, a representative of which we may call $M$. A more detailed justification of the validity of this diagram is given in Ref. 40.

The diagram is periodic with respect to $s$ : events $(s, c t)$ and $(s+L, c t)$ are identical $(L=2 \pi R)$. The world line of $M$ is $M$ 's time axis $c t^{\prime}$, which makes an angle $\delta$ with the axis ct of $C$, where $\tan \delta=v / c$. Obviously, it is a helix winding around the cylinder. The axis $s^{\prime}$ describing events that are simultaneous with $t^{\prime}=0$ from $M^{\prime}$ 's point of view makes the same angle $\delta$ with the $s$ axis, which means that this line of simultaneous events does not close on itself. ${ }^{41}$ It would form a helix, too, if its indefinite continuation were meaningful. We conclude that Einstein synchronization fails when performed 


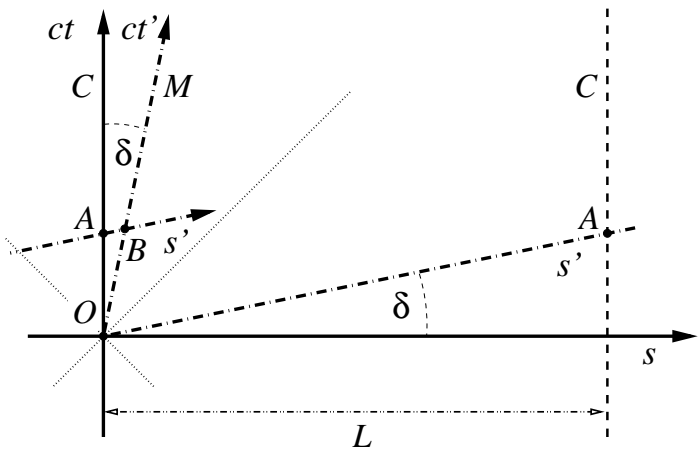

FIG. 1. Minkowski diagram for a rotating circle. The spatial coordinate $s$ is the arclength in an inertial system that is at rest w.r.t. the center of the disk, $s^{\prime}$ the corresponding quantity for corotating disk observers. The direction of rotation is to the right. Dotted lines denote the light cones centered at the intersection of the world lines of $C$ and $M$.

along a path around the full circle. A standard clock on the disk at event $B$ is nominally synchronized with a clock at $O$ and they both show time $t^{\prime}=0$ at these events, respectively. However, $B$ is at the same place as $O$ according to $M$, both events are on $M$ 's world line, but $B$ happens after $t^{\prime}=0$. So the two clocks show different times for event $B$ but claim to be synchronized, which is a contradiction. Hence, Einstein synchronization starting at $O$ (on a path moving to the right) may be extended up to but not including point $B{ }^{42}$

What this means is that a standard clock carried slowly ${ }^{43}$ around the circle in the rotating frame will lag behind one that has remained in place when they meet again. The time lag can be easily calculated from the diagram. Between events $O$ and $A$ in $C$ 's system there is a time difference of $\Delta t_{L}=(L / c) \tan \delta=\left(v / c^{2}\right) L$. Events $A$ and $B$ are simultaneous from $M$ 's point of view and $A$ happens in a system in motion relative to $M$, so the interval $O A$ must be shorter than $O B$ by the time dilation factor. Therefore,

$$
\Delta t_{L}^{\prime}=\gamma \frac{v}{c^{2}} L=2 \pi R \gamma \frac{v}{c^{2}}
$$

A clock carried around the circle in the opposite direction will gain the same time with respect to a clock that stays in place.

We know that in the system described by the primed coordinates, light moves at speed $c$ when time is measured on local clocks. So on these clocks a light ray moving in the corotating direction should take the total time $L^{\prime} / c=\gamma L / c$. This is the time reading on the last of the series of Einstein synchronized clocks, when the light beam returns. But the reading on the clock that stayed in place must be ahead of this by the time gap $\Delta t_{L}^{\prime}$. So the time taken by the light signal is

$$
\Delta t_{+}^{\prime}=\gamma \frac{L}{c}+\gamma \frac{v}{c^{2}} L=\gamma \frac{L}{c}\left(1+\frac{v}{c}\right)=\frac{2 \pi R}{c}\left(\frac{1+\frac{v}{c}}{1-\frac{v}{c}}\right)^{1 / 2}=\tau_{+} .
$$

For a ray sent in the counterrotating direction, the time gap has to be subtracted, so we obtain

$$
\Delta t_{-}^{\prime}=\gamma \frac{L}{c}\left(1-\frac{v}{c}\right)=\frac{2 \pi R}{c}\left(\frac{1-\frac{v}{c}}{1+\frac{v}{c}}\right)^{1 / 2}=\tau_{-} .
$$

Both results agree with Eq. (2), so the average speeds of light computed from these expressions are $c_{ \pm}$from Eq. (3). This is a disk-frame based description leading to the same result as the one inferred by an external observer. Within this description, the speed of light is $c$ everywhere except at the point on the circle where we put the time gap. The position of this point is arbitrary but there must inevitably be such a point. So we can say that in this explanation, while locally the speed of light is measured to be $c$ at any place, the global speed distribution is inhomogeneous with a $\delta$ function behaviour of the inverse speed of light of strength $1 / c_{i}= \pm \Delta t_{L}^{\prime} \delta\left(s^{\prime}-s_{0}\right)$ superimposed on a homogeneous distribution with $1 / c_{h}=1 / c$.

Thus, we have obtained a valid explanation of the Sagnac effect within special relativity using standard Einstein synchronization.

It is then not difficult to resolve Selleri's paradox. In our description, the forward and backward speeds of light are $c$ everywhere except at the position of the time gap. When going to the limit of infinite disk radius, we may conveniently have that gap move to infinity, ${ }^{44}$ so we obtain an inertial system, in which the speed of light is c everywhere, which is what we expect. We may also note that the basic premise of Selleri's argument, requiring the ratio of the forward and backward speeds of light to have the value $q \neq 1$, given in (4), is nowhere satisfied. The reason is that Einstein synchronization breaks the rotational symmetry invoked by Selleri, a fact that should be immediately clear from the Minkowski diagram. Selleri used the symmetry to show that the average velocity of light is equal to its local velocity. Instead, the local speed is $c$ everywhere and the average is modified into $c_{+}$ and $c_{-}$, respectively, due to the time gap, to be added for the evaluation of $c_{+}$and to be subtracted for $c_{-}$.

We might consider the case closed. However, we should not do so without a certain uneasiness. The argument about time dilation made after Eq. (5) does not hold in our solution. Different disk observers at the same radius can synchronize their clocks, with the minor nuisance of the time gap, the crossing of which has to be avoided. But if this is true, Selleri's simple arguments based on rotational symmetry become convincing. There is nothing in the physics of the system that should render them invalid. In fact, this kind of argument is quite fundamental, possibly more fundamental than the second postulate of the theory of relativity! It would definitely be preferable to solve the problem retaining the symmetry. This will be done in the next section, providing some additional insight.

\section{AN ALTERNATIVE SYNCHRONIZATION}

The crucial point is that we are not obliged to use Einstein synchronization. After all, the time gap arising with this approach is awkward. It may be avoided by a different synchronization of clocks along the circle $r=R$.

One way to make the gap disappear is to advance each of the Einstein synchronized clocks along the $s^{\prime}$ axis in Fig. 1 by $\Delta t^{\prime}\left(s^{\prime}\right)=\Delta t_{L}^{\prime} s^{\prime} / L^{\prime}$. Then after a full turn around the circle $\left(s^{\prime}=L^{\prime}\right)$, the local clock at event $B$ will read $t^{*}\left(L^{\prime}\right)=t^{\prime}+\Delta t_{L}^{\prime}$, which is precisely the time the clock that stayed at the origin will also read. The new time may be rewritten as $t^{*}=t^{\prime}+$ $\gamma\left(v / c^{2}\right) L s^{\prime} / \gamma L=t^{\prime}+\left(v / c^{2}\right) s^{\prime}$. The transformations between $C^{\prime}$ 's and $M$ 's frames of reference according to the Minkowski diagram of Fig. 1 are the usual Lorentz transformations

$$
\begin{aligned}
t^{\prime} & =\gamma\left(t-\frac{v}{c^{2}} s\right), & s^{\prime} & =\gamma(s-v t), \\
t & =\gamma\left(t^{\prime}+\frac{v}{c^{2}} s^{\prime}\right), & s & =\gamma\left(s^{\prime}+v t^{\prime}\right) .
\end{aligned}
$$


Introducing the gapless synchronization with time $t^{*}$ we have

$$
t^{*}=\frac{t}{\gamma}, \quad s^{*}=s^{\prime}=\gamma(s-v t),
$$

exhibiting that simultaneity is identical for times $t$ and $t^{*}$ $\left(\mathrm{d} t=0\right.$ implies $\mathrm{d} t^{*}=0$ and vice versa). The same synchronization may be obtained in a variety of more practical ways as discussed by Cranor et al. ${ }^{45}$ If clocks on the circle $r=R$ are all set to the same time when a spherical light wave sent out from the disk center reaches them and run at their proper rates afterwards, their displayed times will obviously agree with each other for all times according to $C$ (even though they run more slowly than $C$ 's clock due to time dilation). Alternatively, clocks may be synchronized by the Einstein procedure before the disk rotates, without being reset after the disk has reached constant angular velocity. Because all clocks suffer the same acceleration program and hence have the same velocity with respect to a central observer, they will remain synchronized from his (and $C$ 's) point of view. We shall call this alternative way of establishing simultaneity central synchronization, for obvious reasons.

Let us calculate the velocity of light tangential to the circle as measured using centrally synchronized clocks. Setting $\mathrm{d} s / \mathrm{d} t= \pm c$, we find from (11)

$$
\frac{\mathrm{d} s^{*}}{\mathrm{~d} t^{*}}=\gamma^{2} \frac{\mathrm{d} s-v \mathrm{~d} t}{\mathrm{~d} t}=\gamma^{2}( \pm c-v)=\frac{ \pm c}{1 \pm v / c}= \pm c_{ \pm},
$$

showing that the local speeds are given by $c_{+}$and $c_{-}$from Eq. (3) everywhere along the circle. This is the situation envisioned by Selleri, the velocity of light being homogeneous along the entire circle but different in the forward and backward directions. Since these velocities are the same as in (3), the times taken for the round trip are $\tau_{ \pm}$from (2). Therefore, the Sagnac effect is correctly described with central synchronization, and this description is simpler than the one based on Einstein synchronization, because no singularity appears. Tangential light velocities are uniform everywhere along the circumference of the circle, in full agreement with general symmetry considerations.

Of course, the second postulate of special relativity still holds when read as referring to two-way velocities. On the other hand, its interpretation as a statement about one-way velocities implies Einstein synchronization as well as a restriction of the utility of the formalism, precluding the simplest approach to the description of rotating systems. Moreover, assuming a universal speed of light before discussing synchronization and simultaneity is logically doubtful,, 39 to say the least. The very definition of a velocity requires a definition of simultaneity at different points in space. Einstein himself was quite clear about this. He emphasized that simultaneity of events at spatially separated points $A$ and $B$ is established by definition ${ }^{46}$ Furthermore, he insisted that the constancy of the one-way speed of light is "neither a supposition nor a hypothesis about the physical nature of light, but a stipulation" 47 Therefore, alternative conventions about simultaneity are possible. They may lead to non-universal one-way speeds of light, but that is no problem for special relativity.

Getting back to Selleri's paradox, we notice that with central synchronization there is nothing left to contradict his argument. The ratio of the two speeds of light is preserved on increasing the circle. The limiting case is an inertial system, in which the speed of light is $c_{ \pm}=c /\left(1 \pm \frac{v}{c}\right)$ in the forward and backward directions. But this simply means that the limit process does not change the synchronization from central to Einstein. This is not too surprising, given that at every finite radius, central synchronization preserves simultaneity between the central observer and an observer on the circle. For continuity reasons, the synchronization arising in the limit $R \rightarrow \infty$ will still preserve simultaneity between the two observers, who are then both inertial. We may immediately write down the appropriate transformation laws by simply replacing the arc lengths with the abscissae of the systems:

$$
\begin{aligned}
t^{*} & =\frac{t}{\gamma}, \quad x^{*}=\gamma(x-v t), \\
t & =\gamma t^{*}, \quad x=\frac{1}{\gamma}\left(x^{*}+\gamma^{2} v t^{*}\right) .
\end{aligned}
$$

These are obviously not the Lorentz transformations. But they are equivalent to the Lorentz transformations, as can be seen by setting

$$
x^{\prime}=x^{*}, \quad t^{\prime}=t^{*}-\frac{v}{c^{2}} x^{\prime},
$$

If in the system $S$ described by coordinates $x$ and $c t$ (which is the inertial system of $C$ ) Einstein synchronization is used, the speed of light is isotropic there. Then the coordinates $x^{\prime}$ and $c t^{\prime}$ describe an inertial system $S^{\prime}$, in which this is also true, since they are related via Lorentz transformations to $x, c t$. The coordinates $x^{*}$ and $c t^{*}$ describe the same inertial system $S^{\prime}$, but with clocks at position $x^{\prime}$ advanced by $v x^{\prime} / c^{2}$, i.e., with a different synchronization. Due to this different synchronization, the speed of light is no longer isotropic, albeit the two-way speed of light remains $c$ in all directions as can be shown quite generally ${ }^{48}$ (we have shown it only for round trips aligned with the $s^{\prime}$ or $x^{\prime}$ directions). That this synchronization is legitimate and does not lead to problems with causality can be immediately seen from the fact that $t^{*}$ is, up to a constant factor, equal to the time in $S$, which by assumption allows correct time ordering of events.

Formally, the description of $S^{\prime}$ via $x^{*}, c t^{*}$ has a certain air of Lorentzian ether theory (with $S$ being the absolute rest frame), and it is known that phenomenologically there is no difference between the predictions of Lorentzian ether theory and special relativity ${ }^{49}$ Nevertheless, since we can take any inertial system to be $S$ and any other inertial system to be $S^{\prime}$, it is clear that neither absolute time nor space are involved. Synchronization rather is a matter of choice of the time coordinate. ${ }^{50}$

So the solution to Selleri's paradox is simply this: indeed the speed of light is anisotropic in the limiting inertial system $S^{\prime}$, obtained by letting $R \rightarrow \infty$ with $\omega R=v$ fixed. But this is only the consequence of a specific choice of synchronization, surviving the limit. While central synchronization is very convenient in describing the rotating frame of reference, i.e., at any finite $R$, because it avoids the appearance of a time gap, it is much less useful in the limiting case, where the time gap escapes to infinity anyway and where the non-orthogonality of the time coordinate $t^{*}$ leads to an undesirable asymmetry between inertial systems. Nevertheless, it may be used in that limiting case, too. No contradictions with special relativity arise. All one has to be aware of is that the one-way speed of light is synchronization dependent and that therefore its being different from $c$ does not constitute a problem. The roundtrip speed of light still is $c$, from which it can be proven ${ }^{39}$ that a resynchronization à la Einstein is possible and will lead to the standard formulation of special relativity. 


\section{CONCLUSIONS}

A few final remarks may be appropriate. The main purpose of this article was to shed some light on Selleri's paradox. In particular, I wanted to show that Selleri's argument about rotational symmetry is legitimate (after one has convinced oneself that a common time may be introduced for observers at a fixed distance from the disk center). Even his result on light speeds may be accepted. However, his construction fails to demonstrate a flaw in relativity, because there is some liberty in the choice of synchronization and, hence, the definition of simultaneity, in any theory where there is a maximum speed for causal connections. ${ }^{51}$ It appears that this liberty is unfamiliar to many physicists who were taught the theory via the standard approach, but it is well-known to an informed community $48,51-55$

Most likely, Selleri was not unaware of the reason why the speeds $c_{ \pm}$survive the limit $R \rightarrow \infty$ leading to an inertial system: his requirement of the local speed of light being equal to its average speed enforces a particular synchronization in the rotating frame. (It enforces the synchronization that is compatible with the rotational symmetry of the system.) However, he took this as a sign that "nature" preferred this synchronization. That conclusion is definitely too far-reaching.

In fact, Selleri's symmetry argument is a double-edged sword. It may be turned around to establish isotropy of the speed of light in the inertial frame $S$ of the disk center. From a space-time diagram similar to Fig. 1) we would conclude that rotational symmetry implies a simultaneity-preserving synchronization in $S^{\prime}$, i.e., we must have $\mathrm{d} t^{*}=a(v) \mathrm{d} t$ with $a(v)$ some coefficient depending on the velocity of $S^{\prime}$ in $S$ only ${ }^{56}$ Moreover, the fact that the disk rotates at an angular frequency $\omega=v / R$ gives us a relationship of the type $x^{*}=b(v)(x-v t)+x_{0}$ with coordinate independent $b(v)$ and constant $x_{0}$. The Sagnac effect yields - experimentally - the ratio $c_{+} / c_{-}=q=(c-v) /(c+v)$ of the forward and backward average speeds of light in $S^{\prime}$, which by rotational symmetry are equal to the local speeds of light. Denoting the corresponding speeds in $S$ by $\tilde{c}_{ \pm}$, we have from $\pm c_{ \pm}=\mathrm{d} x^{*} / \mathrm{d} t^{*}=b(v) a(v)^{-1}(\mathrm{~d} x / \mathrm{d} t-v)$ that $\tilde{c}_{ \pm}= \pm \mathrm{d} x / \mathrm{d} t$ satisfy $\left(\tilde{c}_{+}-v\right) /\left(\tilde{c}_{-}+v\right)=q$. Assuming further the second postulate of special relativity to hold for two-way speeds of light, we get another equation for the two velocities, reading $2 / c=1 / \tilde{c}_{+}+1 / \tilde{c}_{-}$. We then have two (nonlinear) equations for the two quantities $\tilde{c}_{ \pm}$that can be reduced to a quadratic equation for one of them. There are two sets of solutions, of which only $\tilde{c}_{+}=\tilde{c}_{-}=c$ satisfies the requirement of positivity of both speeds.

Now consider a second rotating disk with its center moving at a constant velocity with respect to $S$, thus defining an inertial system $S^{\prime \prime}$. (We might even impose $S^{\prime \prime}=S^{\prime}$, taking $v$ for that velocity.) Using the same symmetry arguments, we can argue that the speed of light is isotropic in $S^{\prime \prime}$ as well, and, by extension, in any inertial system. So Selleri's own arguments seem to imply both isotropy ${ }^{57}$ and anisotropy of the speed of light in some inertial systems, which looks like a contradiction. To avoid it, Selleri would have to invoke that for a disk-center inertial system in which the speed of light is already anisotropic (such as $S^{\prime}$ ), one cannot assume the speed of light on the disk rim to be homogenous, hence it would fail to be equal to its average. In fact, he could assume rotational symmetry only in a single frame (the alleged absolute rest frame). But then it is of course legitimate for any defender of special relativity to drop the assumption of rotational symmetry as well and to go ahead with the arguments described in Sec. III countering Selleri's "proof".

Ironically, it is only within the framework of special relativity that the assumption of rotational symmetry, implying a homogeneous speed of light along the disk rim, can be made for every rotating disk with inertial center, regardless of the translational velocity of that center. Only in special relativity, all these disks are equivalent. Whether the speed of light is isotropic or not in one of the associated limiting inertial systems, then depends on the choice of synchronization made for those systems.

To conclude, the preference for a particular synchronization is one by human decision, not one by nature. Synchronization is conventional. Einstein synchronization is often preferable, just as Cartesian coordinates are in analytic geometry. Situations with specific symmetries may render different coordinate systems preferable in geometry as well as different synchronizations in special relativity.
* Klaus.Kassner@ovgu.de

1 R. J. Kennedy and E. M. Thorndike, "Experimental Establishment of the Relativity of Time," Phys. Rev. 42, 400-418 (1932)

${ }^{2}$ H. E. Ives and G. R. Stilwell, "An Experimental Study of the Rate of a Moving Atomic Clock," J. Opt. Soc. Am. 26, 215-219 (1938)

3 B. Rossi and D. B. Hall, "Variation of the Rate of Decay of Mesotrons with Momentum," Phys. Rev. 59, 223-228 (1941)

4 M. Kaivola, O. Poulsen, E. Riis, and S. A. Lee, "Measurement of the Relativistic Doppler Shift in Neon," Phys. Rev. Lett. 54, 255-258 (1985)

5 S. Reinhardt, G. Saathoff, H. Buhr, L. A. Carlson, A. Wolf, D. Schwalm, S. Karpuk, C. Novotny, G. Huber, M. Zimmermann, R. Holzwarth, T. Udem, T. W. Hänsch, and Gerald Gwinner, "Test of relativistic time dilation with fast optical atomic clocks at different velocities," Nature
Physics 3, 861-864 (2007)

6 J. C. Hafele and R. E. Keating, "Around-the-World Atomic Clocks: Predicted Relativistic Time Gains," Science 177, 166-168 (1972)

7 J. C. Hafele and R. E. Keating, "Around-the-World Atomic Clocks: Observed Relativistic Time Gains," Science 177, 168-170 (1972)

8 J. Bailey, K. Borer, F. Combley, H. Drumm, F. Krienen, F. Lange, E. Picasso, W. von Ruden, F. J. M. Farley, J. H. Field, W. Flegel, and P. M. Hattersley, "Measurements of relativistic time dilation for positive and negative muons in a circular orbit," Nature 268, 301-305 (1977)

9 A. A. Michelson and E. W. Morley, "On the Relative Motion of the Earth and the Luminiferous Ether," Am. J. Sci. 34, 333-345 (1887)

10 A. Brillet and J. L. Hall, "Improved Laser Test of the Isotropy of Space," Phys. Rev. Lett. 42, 549-552 (1979)

11 D. Hils and J. L. Hall, "Improved Kennedy-Thorndike Ex- 
periment to Test Special Relativity," Phys. Rev. Lett. 64, 1697-1700 (1990)

12 V. W. Hughes, H. G. Robinson, and V. Beltran-Lopez, "Upper Limit for the Anisotropy of Inertial Mass from Nuclear Magnetic Resonance," Phys. Rev. Lett. 4, 342-344 (1960)

13 R. W. P. Drever, "A search for anisotropy of inertial mass using a free precession technique," Phil. Mag. 6, 683-687 (1961)

14 V. A. Kostelecký and C. D. Lane, "Constraints on Lorentz violation from clock-comparison experiments," Phys. Rev. D 60, 116010 (1999)

15 M. E. Tobar, P. Wolf, Sébastien Bize, G. Santarelli, and V. Flambaum, "Testing local Lorentz and position invariance and variation of fundamental constants by searching the derivative of the comparison frequency between a cryogenic sapphire oscillator and hydrogen maser," Phys. Rev. D 81, 022003 (2010)

16 D. Mattingly, "Modern Tests of Lorentz Invariance," Living Rev. Relativity 8, 5-84 (2005),

http://www.livingreviews.org/lrr-2005-5, cited 31/5/2012

17 T. Alväger, F. J. Farley, J. Kjellman, and L. Wallin, "Test of the second postulate of special relativity in the $\mathrm{GeV}$ region," Phys. Lett. 12, 260-262 (1964)

18 K. Brecher, "Is the Speed of Light Independent of the Velocity of the Source?." Phys. Rev. Lett. 39, 1051-1054 (1977)

19 M. M. Rogers, A. W. McReynolds, and F. T. Rogers, "A Determination of the Masses and Velocities of Three Radium B Beta-Particles," Phys. Rev. 57, 379-383 (1940)

20 T. Roberts and S. Schleif, "What is the experimental basis of Special Relativity?." Usenet Physics FAQ. University of California, Riverside. (2007), cited 01 June 2012

21 C. Will, "Special Relativity: A Centenary Perspective," in Einstein. Poincaré Seminar 2005, edited by T. Damour, O. Darrigol, B. Duplantier und V. Rivasseau (Birkhäuser Verlag, Basel, 2005) pp. 33-58

${ }^{22}$ E. F. Taylor and J. A. Wheeler, Spacetime Physics: Introduction to Special Relativity, 2nd ed. (W.H. Freeman and Co., New York, 1992)

23 While Boltzmann did have major problems in finding acceptance for his theory, there are hardly as many crackpots believing to be able to refute the foundations of irreversibility as there are disprovers of special relativity.

24 The offside rule in soccer is definitely incompatible with special relativity. It explicitly refers to the position of a player at the moment when the ball is played at a distant location, not at the moment the player receives the ball. The first position is indefinite given that simultaneity of two distant events is involved, the second corresponds to a single well-defined event.

25 J. M. Supplee, "Relativistic buoyancy," Am. J. Phys. 57, 75-77 (1989)

${ }^{26}$ G. E. A. Matsas, "Relativistic Archimedes law for fast moving bodies and the general-relativistic resolution of the "submarine paradox"," Phys. Rev. D 68, 027701 (2003)

27 F. Selleri, "Noninvariant One-Way Speed of Light and Locally Equivalent Reference Frames," Found. Phys. Lett. 10, 73-83 (1997)

28 R. D. Klauber, "Comments regarding recent articles on relativistically rotating frames," Am. J. Phys. 67, 158-159 (1998)

29 F. Selleri, "Sagnac effect: end of the mystery," in Relativity in rotating frames (Kluwer Academic Publishers, Dordrecht, 2004) pp. 57-78
30 A. Einstein, "Die Grundlage der allgemeinen Relativitätstheorie," Ann. Phys. 354, 769-822 (1916), English Translation in The Principle of Relativity (Methuen, 1923, Reprinted by Dover Publications, New York, 1952), pp. $109-164$.

$31 \varnothing$. Grøn, "Relativistic description of a rotating disk," Am. J. Phys. 43, 869-876 (1975)

${ }^{32}$ K. Kassner, "Spatial geometry of the rotating disk and its non-rotating counterpart," Am. J. Phys. 80 (2012), accepted for publication

33 G. Sagnac, "L'éther lumineux démontré par l'effet du vent relatif d'éther dans un interféromètre en rotation uniforme," Compt. Rend. Acad. Sci. 157, 708-710 (1913), English translation in Relativity in rotating frames, eds. G. Rizzi and M. L. Ruggiero, (Kluwer Academic Publishers, Dordrecht, 2004), ch. 2, pp. 5-7

34 This can be tested by exchanging light signals between them and verifying that for any given pair of observers $O_{1}$, $\mathrm{O}_{2}$ these will always take the same time on the clock of $O_{1}$ $\left(\mathrm{O}_{2}\right)$ to move from $\mathrm{O}_{1}$ to $\mathrm{O}_{2}\left(\mathrm{O}_{2}\right.$ to $\left.\mathrm{O}_{1}\right)$ and back.

35 F. Selleri, "Noninvariant One-Way Velocity of Light," Found. Phys. 26, 641-664 (1996)

36 This formulation does not properly take into account that the same laws may be given in different mathematical forms.

37 Selleri believes 35 the second postulate to be a consequence of the first, which it clearly is not, in a proper formulation.

38 To Einstein synchronize clock $B$ with clock $A$, send a light signal from $B$ to $A$ to be returned immediately with the clock reading of $A$. Then set the time on $B$ to the time reading from $A$ plus half the time span elapsed on $B$ between emission and reception of the signal.

39 E. Minguzzi and A. Macdonald, "Universal One-Way Light Speed from a Universal Light Speed over Closed Paths," Found. Phys. Lett. 16, 593-604 (2003)

40 O. Wucknitz, "Sagnac effect, twin paradox and space-time topology - Time and length in rotating systems and closed Minkowski space-times," (2004), gr-qc/0403111v1

41 G. Rizzi and A. Tartaglia, "Speed of Light on Rotating Platforms," Found. Phys. 28, 1663-1683 (1998)

42 Having established a synchronization scheme for all observers between $O$ and $B$, we may exclude time dilation effects of these observers with respect to each other.

43 So that time dilation effects due to the motion with respect to local disk observers on the circle $r=R$ are negligible.

44 In this limit, the size of the time gap also tends to infinity.

45 M. B. Cranor, E. M. Heider, and R. H. Price, "A circular twin paradox," Am. J. Phys. 68, 1016-1020 (1999)

46 A. Einstein, "Zur Elektrodynamik bewegter Körper," Ann. Phys. 322, 891-921 (1905), English translation in The Principle of Relativity (Methuen, 1923, reprinted by Dover Publications, New York, 1952), pp. 35 - 65, "On the electrodynamics of moving bodies".

47 A. Einstein, Relativity: The Special and the General Theory, $15^{\text {th }}$ ed. (Crown, New York, 1952)

48 R. Anderson, I. Vetharaniam, and G. E. Stedman, "Conventionality of Synchronisation, Gauge Dependence and Test Theories of Relativity," Phys. Rep. 295, 93-180 (1998)

49 D. Bohm, The theory of relativity (W. A. Benjamin, Inc., New York, 1965) reprinted by Routledge, Oxon, UK, 1996

$50 t^{*}$ is the time coordinate of a non-orthogonal frame $\left(c t^{*}, x^{*}\right)$, while $t^{\prime}$ and $x^{\prime}$ are orthogonal to each other.

51 A. Grünbaum, "Logical and Philosophical Foundations of the Special Theory of Relativity," Am. J. Phys. 23, 450- 
$464(1955)$

${ }^{52}$ H. Reichenbach, Axiomatik der relativistischen Raum-ZeitLehre (Vieweg, Braunschweig, 1924) English translation: Axiomatization of the Theory of Relativity (Univ. California Press, Berkeley, 1969)

${ }^{53}$ R. Mansouri and R. Sexl, "A Test Theory of Special Relativity: I. Simultaneity and Clock Synchronization," Gen. Rel. Grav. 8, 497-513 (1977)

${ }^{54}$ G. Rizzi, M. L. Ruggiero, and A. Serafini, "Synchroniza- tion gauges and the principles of special relativity," Found. Phys. 34, 1835-1887 (2004)

55 Relativity in rotating frames, edited by G. Rizzi and M. L. Ruggiero (Kluwer Academic Publishers, Dordrecht, 2004)

56 Due to time dilation, $a(v)=1 / \gamma(v)$, but we do not have to know this for our reasoning.

57 Since we consider only two dimensions of space-time here, isotropy essentially means equality of the forward and backward speeds of light. Of course, these considerations can be extended to four dimensions and full space-time. 